\title{
Educación Superior y la Formación en Emprendimiento Interdisciplinario: Un Caso de Estudio
}

\author{
Julio C. Contreras-Velásquez, Sandra Y. Wilches-Duran, Modesto E. Graterol-Rivas y María J. \\ Bautista-Sandoval \\ Universidad Simón Bolívar sede Cúcuta, Colombia. Grupos de investigación: Gestión de Innovación y \\ Emprendimiento; Altos Estudios de Frontera (ALEF). Avenida 3 \# 13-34 Barrio La Playa, Cúcuta- \\ Colombia (e-mail: j.contrerasv@unisimonbolivar.edu.co; swilches@unisimonbolivar.edu.co; \\ m.graterol@unisimonbolivar.edu.co; m.bautista@unisimonbolivar.edu.co)
}

Recibido Dic. 5, 2016; Aceptado Ene. 20, 2017; Versión final Feb. 10, 2017, Publicado Jun. 2017

\begin{abstract}
Resumen
La investigación que se presenta analiza desde el paradigma positivista deductivo-lógico, la relación entre la interdisciplinariedad de equipos emprendedores, y las evaluaciones sobre innovación e impacto en la sociedad de ideas de negocio propuestas por una muestra de 540 estudiantes universitarios agrupados en 116 equipos (formados por 4 o 5 estudiantes). Se calcula el Índice de Variación Cualitativa (IQV) para determinar el nivel de interdisciplinariedad de los equipos. Para el contraste de hipótesis de asociación entre las variables independientes y dependientes se emplea análisis de varianza (ANOVA). Después de confirmar la existencia de diferencias significativas, se aplica la prueba Post-hoc para establecer los niveles donde se hallaban dichas diferencias. Se concluye que el promedio de la evaluación obtenido en innovación y el impacto social de la ideas de negocios es significativamente mayor en los grupos con mayor nivel de interdisciplinariedad, que en los conformados por estudiantes de la misma disciplina.
\end{abstract}

Palabras clave: emprendimiento; educación superior; interdisciplinario; idea de negocio

\section{Higher Education and Training in Interdisciplinary Entrepreneurship: A Study Case}

\begin{abstract}
This research analyzes from a deductive-logical positivist paradigm, the relationship between interdisciplinary of teams of entrepreneurs and the evaluation of innovation and impact on society of business ideas proposed by a sample of 540 university students grouped in 116 teams (formed by 4 or 5 students). Index of Qualitative Variation (IQV) is calculated to determine the level of interdisciplinary teams. To test the hypothesis of association between the independent variable and dependent variable the statistical technique analysis of variance (ANOVA) was used. After checking that there are significant differences the Post-hoc multiple comparisons test is applied, to determine the levels where such differences occurred. It is concluded that average evaluation obtained in innovation and the impact on society of the business ideas is significantly higher in the groups with higher level of interdisciplinary, than in those that are conformed by students of the same discipline.
\end{abstract}

Keywords: entrepreneurship; higher education; interdisciplinary; business idea 


\section{INTRODUCCIÓN}

El crecimiento económico de un país depende del número y calidad de sus empresas, ya que estas inciden en la generación de empleo, desarrollo regional e innovación de productos y servicios (Bucardo et al., 2015); por esto es necesario analizar a los emprendedores que las crean, quienes son considerados agentes facilitadores de la sostenibilidad humana (Soria-Barreto et al., 2016; Wyness et al., 2015). Ramos y Bayter (2012) plantean que para que el emprendimiento genere innovación, además de empleo e ingresos, se requiere la integración de la universidad, Estado, comunidad y empresa, para crear escenarios cooperativos que conformen alternativas productivas y fomenten la cultura emprendedora, fundamentada en el desarrollo de competencias creativas y socialmente responsables para crear nuevo valor en los modelos de negocio.

El fomento y la difusión de la cultura emprendedora universitaria se ha convertido en elemento clave para generar impacto social y económico, a través de los egresados (Sánchez y Ros, 2014), sin embargo, algunos de ellos tienen dificultad para iniciar empresas sostenibles y acceder al campo laboral, siendo así cuestionada la formación orientada a estudiantes para el entorno empresarial (Priegue et al., 2014). Esto plantea a las universidades, el reto de diseñar cursos académicos de emprendimiento utilizando pedagogías aplicadas en ambientes similares al empresarial (Seikkula-Leino et al., 2015; Solomon, 2007).

Según Carvajal (2010), la globalización ha llevado a las organizaciones a desarrollar sistemáticamente su actividad económica con equipos de trabajo interdisciplinares con el propósito de desarrollar el objetivo común de la organización. En este sentido, la investigación analiza la relación entre la interdisciplinariedad, y el grado de innovación e impacto social de ideas de negocio propuestas por una muestra de 540 estudiantes, agrupados en 116 equipos, pertenecientes a la Universidad Simón Bolívar Sede Cúcuta. Para establecer los niveles de interdisciplinariedad, se tiene en cuenta la heterogeneidad disciplinar o programa académico de procedencia de los estudiantes de los equipos, cuantificando la variabilidad intra-equipo mediante el Índice De Variación Cualitativa (IQV). La investigación plantea la siguiente hipótesis: A mayor nivel de interdisciplinariedad, el promedio obtenido de la evaluación innovación de la idea de negocio y su impacto social también resulta mayor. Para el contraste de hipótesis de asociación entre la variable independiente y las dependientes, se empleó el análisis de la varianza (ANOVA). Después de comprobar diferencias significativas se realizó la prueba Post-hoc de comparaciones múltiples para determinar los niveles donde se encuentran dichas diferencias.

\section{LA UNIVERSIDAD Y LA FORMACIÓN EN EMPRENDIMIENTO}

La nueva economía del conocimiento se encuentra en un medio altamente competitivo, y en un mundo de cambios constantes que exigen, además de una formación sólida y consistente en competencias generales y específicas, un desarrollo de competencias de empleabilidad que favorezcan la integración a esta realidad económica mundial (Bernal et al., 2014). Es así como, el contexto económico internacional y la dinámica nacional, exigen que las instituciones de educación superior formen profesionales emprendedores, alineados con el reconocimiento de los planes locales, regionales y nacionales, para concretar las políticas, lineamientos y estrategias de inversión asertiva de recursos, en pro del desarrollo regional y la transferencia efectiva de conocimiento tecnocientífico a la sociedad, para crear valor agregado, riqueza social y mejorar la vida de los ciudadanos (Terán y León, 2010).

Según el CONPES (2016), los escenarios formativos y sociales deben fomentar la cultura emprendedora desde sus miembros más jóvenes, para desarrollar en ellos capacidades, responsabilidades y cambios necesarios, para reaccionar con mayor apertura y flexibilidad en el desempeño cotidiano de cualquier actividad profesional. La cultura emprendedora reúne los valores, creencias y aptitudes generalmente compartidas por una sociedad, que sostienen la creencia deseable de un modo de vida, que apoye continuamente la búsqueda de un proceder emprendedor efectivo de los individuos o los grupos (Gibb, 2002). En este sentido, la educación se convierte en facilitadora del emprendimiento, al fomentar la capacidad de los estudiantes para resolver problemas, ser creativos, decidir, comunicar su voluntad, asumir responsabilidades, cooperar, trabajar en red, autoaprender, ser proactivo, tener iniciativa y prepararse para asumir riesgos controlados (Castro et al., 2014; Fawson et al., 2015; Sánchez y Ros, 2014). Para lograr lo anterior, se debe cambiar la enseñanza pasiva y unidireccional por metodologías que involucren al estudiante en la práctica (De la Fuente et al., 2012; lacobucci y Micozzi, 2012; Taatila, 2010).

\section{DISEÑO DE UNA IDEA DE NEGOCIO POR EQUIPOS INTERDISCIPLINARIOS}

La búsqueda de una organización interdisciplinaria, responde a la excesiva especialización de la ciencia contemporánea que fragmenta los problemas de la realidad (García, 1994). En este sentido se hace necesario estudiar los problemas modernos desde varias disciplinas, y conformar equipos para lograr 
soluciones integrales (CEPAL-ONU, 2003; Morin, 2010; Valencia, 2011). Sin embargo, es frecuente encontrar organizaciones que trabajan como islas, en las que se dificulta el trabajo en equipo por parte de sus empleados.

Lo anterior sirve de base para que en el año 2015 se rediseñe el Programa de Formación en Emprendimiento de la Universidad Simón Bolívar Sede Cúcuta, pasando de un modelo de asignaturas curriculares exclusivo para estudiantes de pregrado en Administración de Empresas, a un programa de formación transversal aplicable en los cinco programas académicos de pregrado de la universidad: administración de empresas, comercio y negocios internacionales, derecho, ingeniería sistemas, psicología y trabajo social, adoptando un aprendizaje holístico e integrado que permite que los participantes se beneficien del trabajo colaborativo interdisciplinar (Álvarez, 2004; Gimmon, 2014; Rodríguez et al., 2013; Vicens y Grullón, 2011; Wood, 1999).

El programa de formación en emprendimiento está distribuido en tres módulos, cada uno con intensidad de 16 horas presenciales y son orientados por mentores de diferentes disciplinas, expertos en negocios, como lo sugiere Li et al. (2003). Después de terminar el tercer módulo, los estudiantes deben presentar su idea de negocio ante mínimo tres jurados expertos en emprendimiento e innovación, que evalúan las propuestas, según cinco criterios que tienen una ponderación asignada según el nivel de importancia. Las ideas de negocio que obtienen un valor superior a siete en la evaluación siguen al módulo cuatro, que consiste en un acompañamiento por mentores especializados en diferentes disciplinas por un período de dos años. En esta fase se elabora el plan de negocio y se buscan recursos financieros para iniciar la operación de la empresa.

\section{ANÁLISIS DE RESULTADOS SOBRE LA INTERDISCIPLINARIEDAD}

La población de estudio son los 669 estudiantes que han finalizado el módulo tres y su idea de negocio ha sido evaluada (Ver Tabla 1). La muestra está formada por 540 sujetos, estudiantes que proponen ideas de negocio de emprendimiento distribuidos en 116 equipos conformados por más de dos estudiantes.

Tabla 1: Cantidad estudiantes en formación de emprendimiento por módulo (Período 2015-1 a 2016-1)

\begin{tabular}{|c|c|c|c|c|}
\hline \multirow{2}{*}{ Módulo } & \multicolumn{3}{|c|}{ Período académico } & \multirow{2}{*}{ Total } \\
\cline { 2 - 4 } & $2015-1$ & $2015-2$ & $2016-1$ & \\
\hline 1 & 667 & 470 & 574 & 1711 \\
\hline 2 & 297 & 501 & 362 & 1160 \\
\hline 3 & 0 & 243 & 426 & 669 \\
\hline Total & 964 & 1214 & 1362 & 3540 \\
\hline
\end{tabular}

Para determinar los niveles de interdisciplinariedad, definida en este caso de estudio como la heterogeneidad de los equipos de trabajo, se hace necesario analizar la variabilidad intra-equipo, utilizando el estadístico Índice de Variación Cualitativa (IQV). Se obtiene a través de la Ecuación 1, donde $k$ es el número de categorías dentro de cada grupo de la variable y Pct, la frecuencia relativa asociada a cada una de ellas:

$I Q V=\frac{K\left(100^{2}-\sum P c t^{2}\right)}{100^{2}(K-1)}$

EI IQV varía entre valores de 0 a 1 . Será cercano a cero, cuando la mayoría de los casos caen en una categoría, es decir existe, dispersión =0, (homogeneidad) o en este caso, no existe interdisciplinariedad, mientras que más se acerca a su valor máximo $=1$, alta dispersión (heterogeneidad) mayor es la interdisciplinariedad en los equipos. En la Tabla 2 se muestra el valor obtenido en cada equipo como resultado de la evaluación promedio en las variables innovación e impacto en la sociedad, así como el IQV.

Se realiza un análisis de correlación a fin de cuantificar el grado de relación lineal existente entre las variables y medir el grado de ajuste de la nube de puntos a una recta, para esto analizamos las gráficas de dispersión a fin de determinar el grado de ajuste lineal. En el gráfico de dispersión entre las variables evaluación promedio de innovación y la evaluación promedio del impacto en la sociedad de la idea de negocio (Fig. 1) se observa que existe patrón lineal, es decir, los datos se ajustan a una recta, por tanto se comprueba el supuesto de linealidad; por tanto para medir esta relación determínanos el coeficiente de correlación de Pearson. 
Tabla 2: Evaluación promedio obtenida e IQV por equipo de emprendedores

\begin{tabular}{lccl}
\hline Equipo & Impacto & Innovación & IVC \\
\hline Equipo 001 & 4.7 & 5.3 & 0.00000 \\
Equipo 002 & 6.0 & 6.0 & .96300 \\
Equipo 003 & 7.0 & 6.0 & .88890 \\
Equipo 004 & 7.5 & 7.5 & .93750 \\
Equipo 005 & 5.5 & 5.0 & 0.00000 \\
Equipo 006 & 4.3 & 4.7 & 0.00000 \\
Equipo 007 & 6.3 & 5.8 & .64000 \\
Equipo 008 & 8.3 & 7.3 & .48980 \\
Equipo 009 & 6.3 & 7.0 & 0.00000 \\
Equipo 010 & 7.7 & 8.7 & .75000 \\
Equipo 011 & 8.7 & 8.7 & .96000 \\
Equipo 012 & 7.0 & 8.5 & .96000 \\
Equipo 013 & 6.0 & 7.0 & .75000 \\
Equipo 014 & 8.3 & 8.0 & .64000 \\
Equipo 015 & 6.0 & 5.7 & .64000 \\
Equipo 016 & 6.0 & 6.0 & 0.00000 \\
Equipo 017 & 5.0 & 5.5 & 1.00000 \\
Equipo 018 & 7.5 & 9.0 & .75000 \\
Equipo 019 & 8.0 & 9.0 & .75000 \\
Equipo 020 & 5.0 & 4.5 & 0.00000 \\
Equipo 021 & 8.0 & 8.0 & .96000 \\
Equipo 022 & 4.5 & 5.0 & .55560 \\
Equipo 023 & 5.0 & 5.0 & .88890 \\
Equipo 024 & 8.0 & 8.3 & 0.00000 \\
Equipo 025 & 6.0 & 6.3 & .96000 \\
Equipo 026 & 7.7 & 7.7 & .96000 \\
Equipo 027 & 7.7 & 8.0 & 1.00000 \\
Equipo 028 & 3.5 & 4.0 & .75000 \\
Equipo 029 & 6.0 & 6.7 & .75000 \\
Equipo 030 & 9.5 & 9.0 & .75000 \\
Equipo 031 & 6.0 & 6.5 & .84000 \\
Equipo 032 & 5.3 & 6.7 & 0.00000 \\
Equipo 033 & 7.3 & 6.7 & .75000 \\
Equipo 034 & 3.0 & 7.0 & .93750 \\
Equipo 035 & 8.0 & 7.5 & 0.00000 \\
Equipo 036 & 5.0 & 8.3 & .84000 \\
Equipo 037 & 7.0 & 8.5 & 0.00000 \\
Equipo 038 & 6.7 & 6.0 & 0.00000 \\
Equipo 039 & 6.0 & 7.3 & 0.00000 \\
Equipo 040 & 8.8 & 6.8 & .96000 \\
Equipo 041 & 4.0 & 4.7 & .75000 \\
Equipo 042 & 2.5 & 1.5 & .88890 \\
Equipo 043 & 4.0 & 6.0 & .93750 \\
Equipo 044 & 6.0 & 8.0 & 0.00000 \\
Equipo 045 & 8.5 & 8.5 & .96000 \\
Equipo 046 & 5.5 & 4.5 & 0.00000 \\
Equipo 047 & 6.5 & 7.0 & 0.00000 \\
Equipo 048 & 8.0 & 9.0 & .93750 \\
Equipo 049 & 6.0 & 6.5 & .93750 \\
Equipo 050 & 7.7 & 8.3 & .88890 \\
Equipo 051 & 5.7 & 6.7 & .55560 \\
Equipo 052 & 7.5 & 6.0 & 0.00000 \\
Equipo 053 & 5.5 & 5.0 & .64000 \\
Equipo 054 & 5.7 & 6.7 & 0.00000 \\
Equipo 055 & 7.3 & 6.3 & 0.00000 \\
Equipo 056 & 8.0 & 8.0 & .75000 \\
Equipo 057 & 7.0 & 7.0 & .96000 \\
EQUIPO 058 & 6.0 & 5.7 & .91840 \\
\hline & & & \\
\hline
\end{tabular}

\begin{tabular}{lccl}
\hline Equipo & Impacto & Innovación & IVC \\
\hline Equipo 059 & 8.0 & 8.0 & .93750 \\
Equipo 060 & 6.3 & 6.0 & .84000 \\
Equipo 061 & 5.5 & 5.5 & .75000 \\
Equipo 062 & 6.7 & 6.7 & 0.00000 \\
Equipo 063 & 5.5 & 7.5 & .88890 \\
Equipo 064 & 7.7 & 6.7 & .84000 \\
Equipo 065 & 7.0 & 6.0 & .75000 \\
Equipo 066 & 5.3 & 5.7 & .88890 \\
Equipo 067 & 9.0 & 3.5 & 0.00000 \\
Equipo 068 & 7.5 & 7.0 & .96000 \\
Equipo 069 & 7.3 & 7.0 & .64000 \\
Equipo 070 & 4.0 & 6.0 & 0.00000 \\
Equipo 071 & 7.0 & 6.5 & 0.00000 \\
Equipo 072 & 4.0 & 5.3 & 0.00000 \\
Equipo 073 & 4.0 & 4.0 & 0.00000 \\
Equipo 074 & 5.3 & 7.0 & 0.00000 \\
Equipo 075 & 8.0 & 8.0 & 0.00000 \\
Equipo 076 & 7.5 & 5.5 & 0.00000 \\
Equipo 077 & 5.5 & 7.5 & .75000 \\
Equipo 078 & 6.0 & 6.0 & .96000 \\
Equipo 079 & 7.0 & 8.0 & .91670 \\
Equipo 080 & 6.0 & 4.3 & 0.00000 \\
Equipo 081 & 7.3 & 8.7 & 1.00000 \\
Equipo 082 & 5.0 & 7.3 & 0.00000 \\
Equipo 083 & 6.5 & 7.5 & .64000 \\
Equipo 084 & 8.0 & 6.7 & .75000 \\
Equipo 085 & 8.0 & 8.3 & .75000 \\
Equipo 086 & 5.0 & 6.0 & 0.00000 \\
Equipo 087 & 7.5 & 9.0 & 0.00000 \\
Equipo 088 & 5.3 & 5.3 & .88890 \\
Equipo 089 & 6.3 & 5.7 & .91670 \\
Equipo 090 & 4.0 & 6.5 & 0.00000 \\
Equipo 091 & 4.7 & 4.7 & 0.00000 \\
Equipo 092 & 4.8 & 5.3 & 0.00000 \\
Equipo 093 & 7.7 & 7.7 & .88890 \\
Equipo 094 & 6.5 & 7.5 & .75000 \\
Equipo 095 & 6.3 & 5.7 & 0.00000 \\
Equipo 096 & 5.5 & 4.0 & .75000 \\
Equipo 097 & 6.3 & 6.7 & .64000 \\
Equipo 098 & 7.5 & 8.5 & .75000 \\
Equipo 099 & 6.8 & 7.8 & .84000 \\
Equipo 100 & 6.7 & 6.7 & .91670 \\
Equipo 101 & 7.5 & 7.5 & .96000 \\
Equipo 102 & 5.7 & 5.7 & .93750 \\
Equipo 103 & 2.0 & 3.3 & .93750 \\
Equipo 104 & 7.3 & 7.0 & .97960 \\
Equipo 105 & 5.5 & 5.5 & 0.00000 \\
Equipo 106 & 8.5 & 8.5 & .88890 \\
Equipo 107 & 6.3 & 5.3 & .64000 \\
Equipo 108 & 5.0 & 4.5 & .88890 \\
Equipo 109 & 9.7 & 9.3 & .96300 \\
Equipo 110 & 8.0 & 7.3 & .64000 \\
Equipo 111 & 7.3 & 8.0 & .75000 \\
Equipo 112 & 7.5 & 7.0 & .75000 \\
Equipo 113 & 7.5 & 6.0 & .75000 \\
Equipo 114 & 4.8 & 5.8 & 1.00000 \\
Equipo 115 & 6.5 & 6.5 & .75000 \\
EQUIPO 116 & 6.0 & 6.0 & .88890 \\
\hline & & & \\
\hline
\end{tabular}




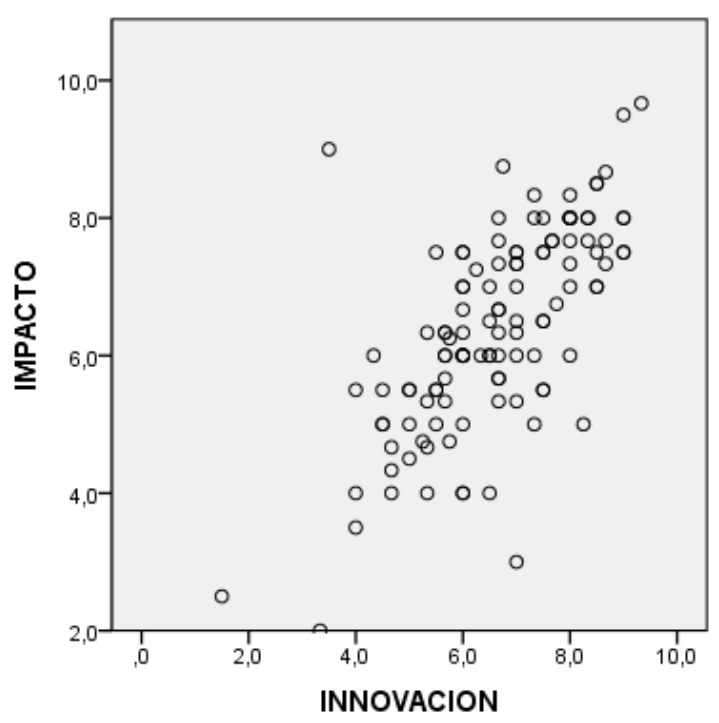

Fig. 1: Gráfico de dispersión entre variables dependientes

El coeficiente de correlación lineal de Pearson (varía entre valores de -1 a 1) $r=0,683$ evidencia una alta correlación lineal positiva entre la evaluación promedio de Innovación de la idea de negocio y la evaluación promedio del Impacto en la sociedad de la idea de negocio, siendo esta relación lineal estadísticamente significativa con (valor de Sig $=.000$, a un nivel de $p=0,01$ ). La Tabla 3 muestra los resultados.

Tabla 3. Correlación entre variables dependientes

\begin{tabular}{|l|l|c|c|}
\hline \multicolumn{2}{|l|}{} & IMPACTO & INNOVACION \\
\hline \multirow{3}{*}{ IMPACTO } & Correlación de Pearson & 1 &, 683 \\
\cline { 2 - 4 } & Sig. (bilateral) & &, 000 \\
\cline { 2 - 4 } & $\mathrm{N}$ & 116 & 116 \\
\hline \multirow{3}{*}{ INNOVACION } & Correlación de Pearson &, 683 & 1 \\
\cline { 2 - 4 } & Sig. (bilateral) &, 000 & \\
\cline { 2 - 4 } & $\mathrm{N}$ & 116 & 116 \\
\hline
\end{tabular}

En el gráfico de dispersión entre las variables evaluación promedio de Innovación de la idea de negocio innovación y el IQV (Ver Fig. 2), al igual que el gráfico de dispersión entre la evaluación promedio del Impacto en la sociedad de la idea de negocio y el IQV (Ver Fig. 3) se puede observar que no existe patrón lineal, los datos no se ajustan a una recta, no cumpliendo el supuesto en este caso; por lo tanto, ejecutamos el equivalente no paramétrico del Correlación de Pearson, el coeficiente de correlación Tau-b de Kendall $\tau_{\mathrm{b}}$ como alternativa que varía entre valores de -1 a 1 .

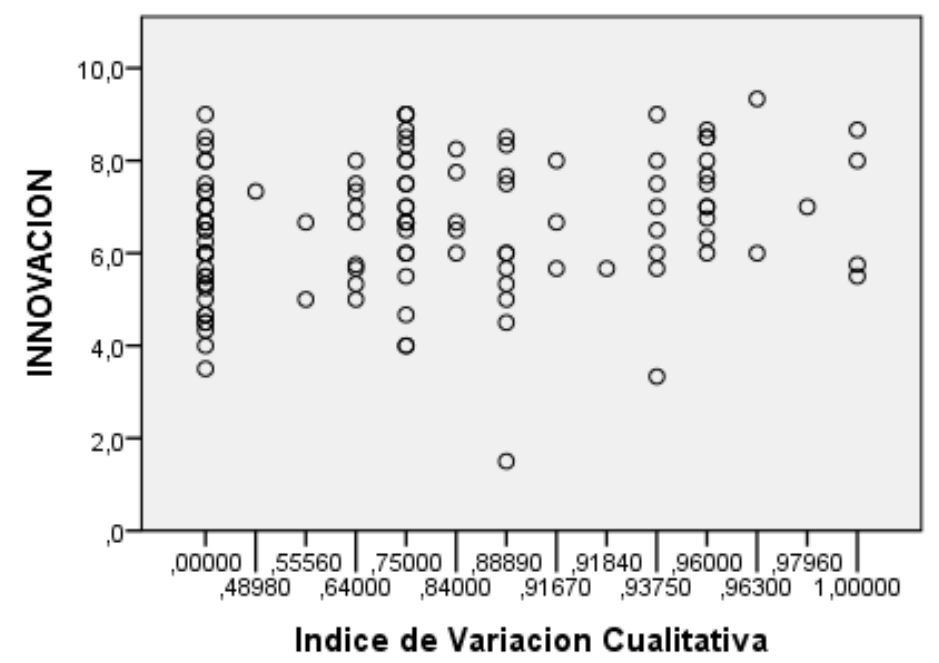

Fig. 2: Gráfico de dispersión entre evaluación promedio en innovación y el IQV 


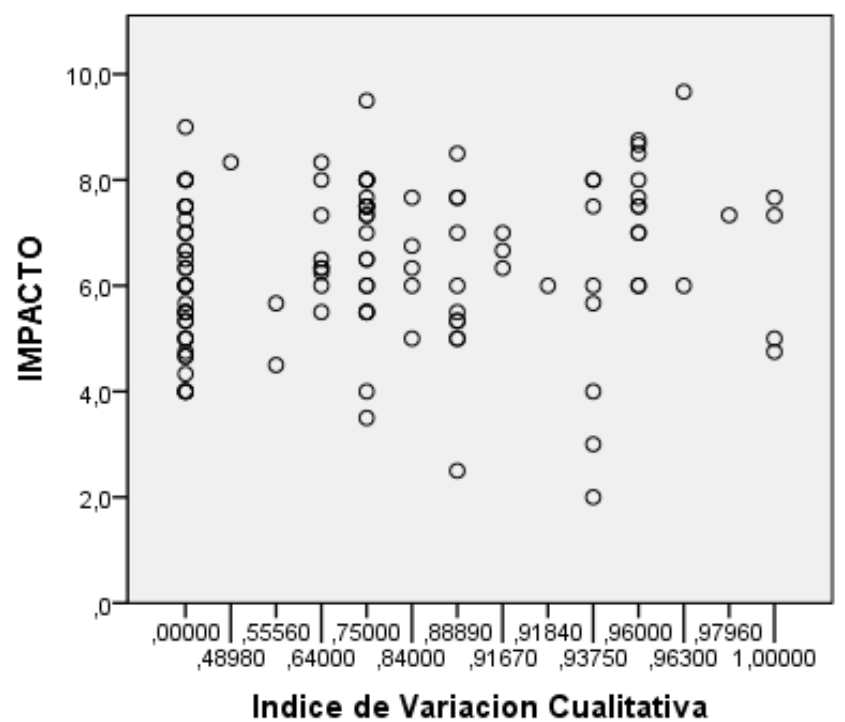

Fig. 3: Gráfico de dispersión entre evaluación promedio en impacto y el IQV

El Coeficiente de correlación Tau-b de correlación de Kendall determina que existe correlación moderada positiva y estadísticamente significativa $(\tau \mathrm{b}=.177, \mathrm{p}=.010)$ a un nivel de $p=.01$ entre la evaluación promedio de Innovación de la idea de negocio y el Índice de Variación Cualitativa. También determina que existe correlación moderada positiva y estadísticamente significativa $(\tau b=.147, p=.032)$ a un nivel de $p$ $=.05$ entre la evaluación promedio del Impacto en la sociedad de la idea de negocio y el Índice de variación Cualitativa. La Tabla 4 muestra los resultados. Los niveles de interdisciplinariedad se establecieron en tres rangos de valores de IQV: "Nivel Alto" entre 0.9 y 1 , "Nivel Medio" entre 0.4 y 0.89 y "Ninguno" entre 0 y 0.39. La Tabla 5 muestra los datos descriptivos del IQV según Nivel de interdisciplinariedad.

Tabla 4: Correlación entre variables dependientes y la independiente. * La correlación es significativa al nivel 0,01 (bilateral). * La correlación es significativa al nivel 0,05 (bilateral)

\begin{tabular}{|c|c|c|c|c|c|}
\hline & & & Innovación & Impacto & $\begin{array}{c}\text { Índice de Variación } \\
\text { Cualitativa }\end{array}$ \\
\hline \multirow{6}{*}{$\begin{array}{l}\text { Tau_b de } \\
\text { Kendall }\end{array}$} & \multirow{2}{*}{ Innovación } & Coeficiente de correlación & 1,000 &, $537^{* *}$ & $177^{* *}$ \\
\hline & & Sig. (bilateral) & . & ,000 & ,010 \\
\hline & \multirow{2}{*}{ Impacto } & Coeficiente de correlación &, $537^{* *}$ & 1,000 & $147^{*}$ \\
\hline & & Sig. (bilateral) & ,000 & . & ,032 \\
\hline & \multirow{2}{*}{$\begin{array}{l}\text { Índice de Variación } \\
\text { Cualitativa }\end{array}$} & Coeficiente de correlación & $177^{* *}$ &, $147^{*}$ & 1,000 \\
\hline & & Sig. (bilateral) & 010 & 032 & . \\
\hline
\end{tabular}

Tabla 5: Índice de Variación Cualitativa clasificado según Nivel de Interdisciplinariedad

\begin{tabular}{|c|c|c|c|c|c|c|c|c|c|}
\hline & & \multicolumn{8}{|c|}{ Nivel de Interdisciplinariedad } \\
\hline & & \multicolumn{2}{|c|}{ Alto } & \multicolumn{2}{|c|}{ Medio } & \multicolumn{2}{|c|}{ Ninguno } & \multicolumn{2}{|c|}{ Total } \\
\hline & & Recuento & $\begin{array}{c}\% \text { de } \mathrm{N} \\
\text { columnas }\end{array}$ & Recuento & $\begin{array}{c}\% \text { de } \mathrm{N} \\
\text { columnas }\end{array}$ & Recuento & $\begin{array}{c}\% \text { de } \mathrm{N} \\
\text { columnas }\end{array}$ & Recuento & $\begin{array}{c}\% \text { de } \mathrm{N} \\
\text { columnas }\end{array}$ \\
\hline \multirow{13}{*}{$\begin{array}{l}\text { Índice de } \\
\text { Variación } \\
\text { Cualitativa }\end{array}$} & ,0000 & 0 & $0,0 \%$ & 0 & $0,0 \%$ & 36 & $100,0 \%$ & 36 & $32,4 \%$ \\
\hline &, 5556 & 0 & $0,0 \%$ & 2 & $4,2 \%$ & 0 & $0,0 \%$ & 2 & $1,8 \%$ \\
\hline & ,6400 & 0 & $0,0 \%$ & 9 & $18,8 \%$ & 0 & $0,0 \%$ & 9 & $8,1 \%$ \\
\hline & ,7500 & 0 & $0,0 \%$ & 22 & $45,8 \%$ & 0 & $0,0 \%$ & 22 & $19,8 \%$ \\
\hline & ,8400 & 0 & $0,0 \%$ & 5 & $10,4 \%$ & 0 & $0,0 \%$ & 5 & $4,5 \%$ \\
\hline & ,8889 & 0 & $0,0 \%$ & 10 & $20,8 \%$ & 0 & $0,0 \%$ & 10 & $9,0 \%$ \\
\hline & ,9167 & 3 & $11,1 \%$ & 0 & $0,0 \%$ & 0 & $0,0 \%$ & 3 & $2,7 \%$ \\
\hline & ,9375 & 6 & $22,2 \%$ & 0 & $0,0 \%$ & 0 & $0,0 \%$ & 6 & $5,4 \%$ \\
\hline & ,9600 & 11 & $40,7 \%$ & 0 & $0,0 \%$ & 0 & $0,0 \%$ & 11 & $9,9 \%$ \\
\hline & ,9630 & 2 & $7,4 \%$ & 0 & $0,0 \%$ & 0 & $0,0 \%$ & 2 & $1,8 \%$ \\
\hline & ,9796 & 1 & $3,7 \%$ & 0 & $0,0 \%$ & 0 & $0,0 \%$ & 1 & $0,9 \%$ \\
\hline & 10,000 & 4 & $14,8 \%$ & 0 & $0,0 \%$ & 0 & $0,0 \%$ & 4 & $3,6 \%$ \\
\hline & Total & 27 & $100,0 \%$ & 48 & $100,0 \%$ & 36 & $100,0 \%$ & 111 & $100,0 \%$ \\
\hline
\end{tabular}


Para someter a comprobación por una parte si existen diferencias significativas entre la valoración de innovación de la idea de negocio y los niveles de interdisciplinariedad de los equipos de trabajo, así como, si existen diferencias significativas entre la valoración de impacto en la sociedad de la idea de negocio y los niveles de interdisciplinariedad de los equipos de trabajo, una vez comprobados los supuestos, se utiliza la técnica estadística del Análisis de la Varianza (ANOVA), donde definimos como variable dependiente o variable resultado, la valoración de innovación y la valoración de impacto en la sociedad de la idea de negocio, buscando determinar en qué medida se puede atribuir la variabilidad de éstas a los niveles de interdisciplinariedad "Nivel Alto", "Nivel Medio" y "Ninguno" definido como variable independiente o predictor. La Tabla 6 muestra los resultados descriptivos de las variables dependientes por Nivel de Interdisciplinariedad.

Tabla 6: Descriptivos variables dependientes por Nivel de Interdisciplinariedad

\begin{tabular}{|c|c|c|c|c|c|c|c|c|c|}
\hline & \multirow{2}{*}{$N$} & \multirow{2}{*}{ Media } & \multirow{2}{*}{$\begin{array}{c}\text { Desviación } \\
\text { típica }\end{array}$} & \multirow{2}{*}{$\begin{array}{l}\text { Error } \\
\text { típico }\end{array}$} & \multicolumn{2}{|c|}{$\begin{array}{c}\text { Intervalo de confianza para } \\
\text { la media al } 95 \%\end{array}$} & \multirow{2}{*}{ Mínimo } & \multirow{2}{*}{ Máximo } \\
\hline & & & & & & $\begin{array}{l}\text { Límite } \\
\text { inferior }\end{array}$ & $\begin{array}{l}\text { Límite } \\
\text { superior }\end{array}$ & & \\
\hline \multirow{4}{*}{ IMPACTO } & Alto & 27 & 7,019 & 13,075 & ,2516 & 6,501 & 7,536 & 4,0 & 9,7 \\
\hline & Medio & 48 & 6,569 & 12,656 & , 1827 & 6,202 & 6,937 & 3,5 & 9,5 \\
\hline & Ninguno & 36 & 5,986 & 13,236 & ,2206 & 5,538 & 6,434 & 4,0 & 9,0 \\
\hline & Total & 111 & 6,489 & 13,417 & , 1274 & 6,237 & 6,742 & 3,5 & 9,7 \\
\hline \multirow{4}{*}{ INNOVACION } & Alto & 27 & 7,228 & 11,481 & ,2210 & 6,774 & 7,683 & 5,5 & 9,3 \\
\hline & Medio & 48 & 6,745 & 13,619 & , 1966 & 6,349 & 7,140 & 4,0 & 9,0 \\
\hline & Ninguno & 36 & 6,167 & 13,368 & ,2228 & 5,714 & 6,619 & 3,5 & 9,0 \\
\hline & Total & 111 & 6,675 & 13,544 & , 1286 & 6,420 & 6,930 & 3,5 & 9,3 \\
\hline
\end{tabular}

\section{Contraste de Hipótesis 1}

Hipótesis nula (Ho): No existen diferencias significativas entre las medias de la valoración de Impacto en la sociedad de la idea de negocio y los Niveles de interdisciplinariedad. Hipótesis alternativa (H1): Existen diferencias significativas entre las medias de la valoración de Impacto en la sociedad de la idea de negocio y los Niveles de interdisciplinariedad. El resultado del ANOVA de la Tabla 7 presenta el valor $F=5,066$, con valor $p$ asociado Sig = 0,008 menor al nivel de significación del .05, por tanto se rechaza la Hipótesis Nula y se acepta la Hipótesis Alternativa $\mathrm{H} 1$, que existen diferencias estadísticamente significativas entre las medias de la valoración de Impacto en la sociedad de la idea de negocio y los Niveles de interdisciplinariedad.

Tabla 7: ANOVA de un factor la valoración de Impacto en la sociedad de la idea de negocio por Nivel de Interdisciplinariedad

\begin{tabular}{lccccc}
\hline & $\begin{array}{c}\text { Suma de } \\
\text { cuadrados }\end{array}$ & gl & $\begin{array}{c}\text { Media } \\
\text { cuadrática }\end{array}$ & F & Sig. \\
\hline Inter-grupos & 16,985 & 2 & 8,493 & 5,066 &, 008 \\
Intra-grupos & 181,044 & 108 & 1,676 & & \\
Total & 198,029 & 110 & & & \\
\hline
\end{tabular}

Una vez comprobado que existen diferencias significativas entre la valoración de impacto en la sociedad de la idea de negocio y los Niveles de interdisciplinariedad, realizamos la pruebas Post hoc de comparaciones múltiples con la finalidad de determinar en qué niveles o grupos se encuentran dichas diferencias. La prueba post hoc de Tukey y Bonferroni (Ver Tabla 8), presenta que existen diferencia significativas entre la valoración de Impacto en la sociedad de la idea de negocio de nivel de Interdisciplinariedad "Alto" y el nivel de interdisciplinariedad "Ninguno" con un valor p asociado Sig de .006, (menor que el nivel de significación de .05) mientras que no hay diferencias significativas entre "Nivel Alto" y "Nivel medio" Sig $=.323>.05$, tampoco entre "Nivel medio" y "Ninguno Sig =.107 >.05. Por tanto, se puede concluir que la valoración de Impacto en la sociedad de la idea de negocio es significativamente mayor en los Niveles de interdisciplinariedad altos que en aquellos grupos con ningún nivel de interdisciplinariedad. 
Tabla 8: Prueba Post hoc de comparaciones múltiples entre Impacto y Nivel de Interdisciplinariedad

\begin{tabular}{|c|c|c|c|c|c|c|c|}
\hline \multicolumn{8}{|c|}{ Variable dependiente: Impacto } \\
\hline & \multirow{2}{*}{$\begin{array}{c}\text { (I) Nivel de } \\
\text { Interdisciplinariedad }\end{array}$} & \multirow{2}{*}{$\begin{array}{c}\text { (J) Nivel de } \\
\text { Interdisciplinariedad }\end{array}$} & \multirow{2}{*}{$\begin{array}{c}\text { Diferencia de } \\
\text { medias (I-J) }\end{array}$} & \multirow{2}{*}{$\begin{array}{l}\text { Error } \\
\text { típico }\end{array}$} & \multirow{2}{*}{ Sig. } & \multicolumn{2}{|c|}{$\begin{array}{c}\text { Intervalo de } \\
\text { confianza al } 95 \%\end{array}$} \\
\hline & & & & & & $\begin{array}{l}\text { Límite } \\
\text { inferior }\end{array}$ & $\begin{array}{l}\text { Límite } \\
\text { superior }\end{array}$ \\
\hline \multirow{6}{*}{$\begin{array}{l}\text { HSD de } \\
\text { Tukey }\end{array}$} & \multirow{2}{*}{ Alto } & Medio & ,4491 & ,3115 & ,323 &,- 291 & 1,189 \\
\hline & & Ninguno & 10,324 & ,3296 & ,006 & ,249 & 1,816 \\
\hline & \multirow{2}{*}{ Medio } & Alto &,- 4491 & ,3115 & ,323 & $-1,189$ & 291 \\
\hline & & Ninguno & ,5833 & ,2855 & ,107 &,- 095 & 1,262 \\
\hline & \multirow{2}{*}{ Ninguno } & Alto & $-10,324$ & ,3296 & ,006 & $-1,816$ &,- 249 \\
\hline & & Medio &,- 5833 & ,2855 & ,107 & $-1,262$ & ,095 \\
\hline \multirow{6}{*}{ Bonferroni } & \multirow{2}{*}{ Alto } & Medio & ,4491 & ,3115 & ,457 &,- 308 & 1,207 \\
\hline & & Ninguno & 10,324 & ,3296 & ,007 & ,231 & 1,834 \\
\hline & \multirow{2}{*}{ Medio } & Alto &,- 4491 & ,3115 & ,457 & $-1,207$ & ,308 \\
\hline & & Ninguno & ,5833 & ,2855 &, 130 &,- 111 & 1,278 \\
\hline & \multirow{2}{*}{ Ninguno } & Alto & $-10,324$ & ,3296 & ,007 & $-1,834$ &,- 231 \\
\hline & & Medio &,- 5833 & ,2855 & ,130 & $-1,278$ &, 111 \\
\hline
\end{tabular}

\section{Contraste de Hipótesis 2}

Hipótesis nula (Ho): No existen diferencias significativas entre las medias de la valoración de Innovación de la idea de negocio y los Niveles de interdisciplinariedad. Hipótesis alternativa (H2): Existen diferencias significativas entre las medias de la valoración de Innovación de la idea de negocio y los Niveles de interdisciplinariedad. El resultado del ANOVA (Ver Tabla 9), presenta el valor $F=5,226$, con valor $p$ asociado Sig = 0,007 menor al nivel de significación del .05, por tanto se rechaza la Hipótesis Nula y se acepta la Hipótesis $\mathrm{H} 2$ de que existen diferencias estadísticamente significativas entre las medias de la valoración de Innovación de la idea de negocio y los Niveles de interdisciplinariedad.

Tabla 9: ANOVA de un factor la valoración de innovación de la idea de negocio por Nivel de Interdisciplinariedad

\begin{tabular}{|l|c|c|c|c|c|}
\hline \multicolumn{7}{|c|}{ INNOVACION } \\
\hline & $\begin{array}{c}\text { Suma de } \\
\text { cuadrados }\end{array}$ & gl & $\begin{array}{c}\text { Media } \\
\text { cuadrática }\end{array}$ & F & Sig. \\
\hline Inter-grupos & 17,805 & 2 & 8,902 & 5,226 &, 007 \\
\hline Intra-grupos & 183,986 & 108 & 1,704 & & \\
\hline Total & 201,791 & 110 & & & \\
\hline
\end{tabular}

Tabla 10: Prueba Post hoc de comparaciones múltiples entre Innovación y Nivel de Interdisciplinariedad

\begin{tabular}{|c|c|c|c|c|c|c|c|}
\hline \multicolumn{8}{|c|}{ Variable dependiente: Innovación } \\
\hline & \multirow{2}{*}{$\begin{array}{c}\text { (I) Nivel de } \\
\text { Interdisciplinariedad }\end{array}$} & \multirow{2}{*}{$\begin{array}{c}\text { (J) Nivel de } \\
\text { Interdisciplinariedad }\end{array}$} & \multirow{2}{*}{$\begin{array}{c}\text { Diferencia de } \\
\text { medias (I-J) }\end{array}$} & \multirow{2}{*}{$\begin{array}{l}\text { Error } \\
\text { típico }\end{array}$} & \multirow{2}{*}{ Sig. } & \multicolumn{2}{|c|}{$\begin{array}{c}\text { Intervalo de } \\
\text { confianza al } 95 \%\end{array}$} \\
\hline & & & & & & $\begin{array}{l}\text { Límite } \\
\text { inferior }\end{array}$ & $\begin{array}{l}\text { Límite } \\
\text { superior }\end{array}$ \\
\hline \multirow{6}{*}{$\begin{array}{l}\text { HSD de } \\
\text { Tukey }\end{array}$} & \multirow{2}{*}{ Alto } & Medio & ,4836 & ,3140 & ,276 &,- 263 & 1,230 \\
\hline & & Ninguno & 10,617 & ,3323 & ,005 & ,272 & 1,851 \\
\hline & \multirow{2}{*}{ Medio } & Alto &,- 4836 & ,3140 & ,276 & $-1,230$ & ,263 \\
\hline & & Ninguno & ,5781 & ,2878 & ,115 &,- 106 & 1,262 \\
\hline & \multirow{2}{*}{ Ninguno } & Alto & $-10,617$ & ,3323 & ,005 & $-1,851$ &,- 272 \\
\hline & & Medio &,- 5781 & ,2878 & ,115 & $-1,262$ & ,106 \\
\hline \multirow{6}{*}{ Bonferroni } & \multirow{2}{*}{ Alto } & Medio & ,4836 & ,3140 & ,379 &,- 280 & 1,247 \\
\hline & & Ninguno & 10,617 & ,3323 & ,005 & ,254 & 1,870 \\
\hline & \multirow{2}{*}{ Medio } & Alto &,- 4836 & ,3140 & ,379 & $-1,247$ & ,280 \\
\hline & & Ninguno & ,5781 & ,2878 & ,141 &,- 122 & 1,278 \\
\hline & \multirow{2}{*}{ Ninguno } & Alto & $-10,617$ & ,3323 & ,005 & $-1,870$ &,- 254 \\
\hline & & Medio &,- 5781 & ,2878 & 141 & $-1,278$ & ,122 \\
\hline
\end{tabular}


Una vez comprobado que existen diferencias significativas entre la valoración de Innovación de la idea de negocio y los Niveles de interdisciplinariedad, realizamos la pruebas Post hoc de comparaciones múltiples con la finalidad de determinar en qué niveles o grupos se encuentran dichas diferencias. Las prueba post hoc de Tukey y Bonferroni presenta que existen diferencias significativas entre valoración de Innovación de la idea de negocio de nivel de Interdisciplinariedad "Alto" y el nivel de interdisciplinariedad "Ninguno" con un valor $p$ asociado Sig de .005, (menor que el nivel de significación de .05) mientras que no hay diferencias significativas entre "Nivel Alto" y "Nivel medio" Sig $=.276>.05$, tampoco entre "Nivel medio" y "Ninguno" Sig $=.115>.05$. La Tabla 10 muestra los resultados. Por tanto se concluye que la valoración de Innovación de la idea de negocio es significativamente mayor en los Niveles de interdisciplinariedad altos que en aquellos grupos con ningún nivel de interdisciplinariedad.

\section{CONCLUSIONES}

El principal hallazgo del estudio es que se aprueban las hipótesis que las evaluaciones en innovación y el impacto social de la idea de negocio son significativamente mayor en los grupos con mayor nivel de interdisciplinariedad, que en los conformados por estudiantes de la misma disciplina, confirmando la necesidad de estudiar los problemas modernos desde varias disciplinas, y conformar equipos para lograr soluciones integrales. También sirve de base para que las universidades diseñen programas de formación en emprendimiento transversal aplicable en todos los programas académicos, adoptando un aprendizaje holístico e integrado que permite que los participantes se beneficien del trabajo colaborativo interdisciplinar.

\section{AGRADECIMIENTOS}

El artículo se vincula al proyecto "Propuesta de programa de formación en emprendimiento y aceleración de negocios para mejorar la cultura emprendedora en Norte de Santander", con código C2011650216, realizado por el grupos de investigación Gestión de Innovación y Emprendimiento; y Altos Estudios de Frontera (ALEF). Los autores agradecen el apoyo financiero por parte de la Universidad Simón Bolívar, sede Cúcuta.

\section{REFERENCIAS}

Álvarez, R. P., Formación superior basada en competencias, interdisciplinariedad y trabajo autónomo del estudiante, Revista iberoamericana de educación: 8, 1-33 (2004)

Bernal, A.; P. Delgado y M. Donoso, Economy of knowledge, Entrepreneurial Culture and Employability in the field of Education. An Approximation to the Spanish Case, Procedia-Social and Behavioral Sciences: 139, 168-174 (2014)

Bucardo, M. A.; M. Saavedra y M. Camarena, Hacia una comprensión de los conceptos de emprendedores y empresarios, Suma de Negocios: 6(13), 98-107 (2015)

Carvajal, Y., Interdisciplinariedad: desafío para la educación superior y la investigación, Luna Azul: (31), 156-169 (2010)

Castro, A.; P. Renés y B. de León, Educación para el emprendimiento: el caso de Cantabria, ProcediaSocial and Behaviioral Sciences: 139, 512-518 (2014)

CEPAL-ONU, Ciencia y tecnología para el desarrollo sostenible. Una perspectiva latinoamericana y caribeña. Impreso en Naciones Unidas, Santiago de Chile (2003)

CONPES, Política nacional de ciencia, tecnología e innovación, 2015-2025, https://goo.gl/EHJqFj, (2016)

De la Fuente, J.; M. Vera y M. Cardelle-Elawar, Aportaciones de la psicología de la innovación y del emprendimiento a la educación, en la sociedad del conocimiento, Electronic Journal of Research in Educational Psychology: 10(28), 941-966 (2012)

Fawson, C.; R. Simmons y R. Yonk, Curricular and programmatic innovation at the intersection of business ethics and entrepreneurship, In The challenges of ethics and entrepreneurship in the global environment, pp 109-130, Emerald Group Publishing Limited (2015)

García, R., Interdisciplinariedad y sistemas complejos, En Ciencias Sociales y Formación Ambiental, pp. 85124, Editorial Gedisa, Barcelona, España (1994) 
Gibb, A., Creating conducive environments for learning and entrepreneurship-living with, dealing with, creating and enjoying uncertainty and complexity, Industry and Higher Education: 16(3), 135-148 (2002)

Gimmon, E., Mentoring as a practical training in higher education of entrepreneurship. Education + Training: 56(8/9), 814-825 (2014)

lacobucci, D. y A. Micozzi, Entrepreneurship education in Italian universities: trend, situation and opportunities, Education + Training: 54(8/9), 673-696 (2012)

Li, J.; Y. Zhang y H. Matlay, Entrepreneurship education in China, Education + Training: 45(8/9), 495-505 (2003)

Morin, E., Sobre la interdisciplinariedad, Publicaciones Icesi: (62), 11-17 (2010)

Priegue, D.; J. García y M. Lorenzo, Higher Education and Enterprising Spirit: The Case of the Universidade de Santiago de Compostela, Procedia Social and Behavioral Sciences: 139, 48-55 (2014)

Ramos, F. S. y L. O. Bayter, Emprendimiento y economía social, oportunidades y efectos en una sociedad en transformación, CIRIEC - España: (75), 129-151 (2012)

Rodríguez, M. y otros cinco autores, Estrategia de ajuste curricular con enfoque interdisciplinario para la farmacología en medicina, Edumecentro: 1(2), 55-61 (2013)

Sánchez, F. y C. Ros, Development of entrepreneurial competence through practicum in pedagogy degree, Procedia-Social and Behavioral Sciences: 139, 116-122 (2014)

Seikkula-Leino, J.; T. Satuvuori, E. Ruskovaara y H. Hannula, How do Finnish teacher educators implement entrepreneurship education? Education+ Training: 57(4), 392-404 (2015)

Solomon, G., An examination of entrepreneurship education in the United States, Journal of small business and enterprise development: 14(2), 168-182 (2007)

Soria-Barreto, K.; S. Zuniga-Jara y S. Ruiz-Campo, Educación e Intención Emprendedora en Estudiantes Universitarios: Un Caso de Estudio, Formación universitaria: 9(1), 25-34 (2016)

Taatila, V. P., Learning entrepreneurship in higher education, Education + Training: 52(1), 48-61 (2010)

Terán, A. y G. León, Visión del emprendimiento desde el Estado y la universidad: El plan de desarrollo de Antioquia visión 2020 con el programa de emprendimiento de la UPB, Revista Ciencias Estratégicas: 18(23), 119-29 (2010)

Valencia, A., Enseñanza del emprendimiento en las facultades de ingeniería, Engineering for a smart planet, innovation, information technology and computational tools for sustainable development, Ninth LACCEI Latin American and Caribbean Conference, Medellín, Colombia, Agosto (2011)

Vicens, L. y S. Grullón, Innovación y emprendimiento: Un modelo basado en el desarrollo del emprendedor, V Foro de Competitividad de las Américas, Santo Domingo, Rep. Dominicana, 5-7 de Octubre (2011)

Wood, G., Interdisciplinary working in built environment education, Education + Training: 41(8), 373-380 (1999)

Wyness, L.; P. Jones y R. Klapper, Sustainability: what the entrepreneurship educators think, Education + Training: 57(8/9), 834-852 (2015) 